\title{
A simple method toward high density SrM dot arrays
}

\author{
A. Kaewrawang, A. Ghasemi, X. Liu, and A. Morisako \\ Spin Device Technology Center, Faculty of Engineering, Shinshu University, 4-17-1 Wakasato, Nagano 380-8553, Japan
}

Strontium ferrite thin films have been deposited on Corning glass with Au underlayer by using DC magnetron sputtering system. Au island structure can be formed on Corning glass by heating the substrate over $100{ }^{\circ} \mathrm{C}$. In order to achieve higher $\mathrm{Au}$ dot density and consequently higher density of SrM, an attempt to decreasing Au thickness has been carried out. The maximum of coercivity and remanent squareness ratio in perpendicular direction are almost constant at $2.3 \mathrm{kOe}$ and 0.45 , respectively, at Au underlayer thickness from $4 \mathrm{~nm}$ to $8 \mathrm{~nm}$. The magnetic interaction between SrM grains prepared on Au nano-dots is less than that for the continuous film.

Key words: Au nano-dots, strontium ferrite, self-assembly, patterned media

\section{Introduction}

The areal density of hard disk drives continues to increase, so there has been a trend to decrease the grain size. However, the trend will be limited by thermal instability or superparamagnetism of the grains in medium. Significant research efforts have been expending in improving media to reduce thermal instability and in designing alternative methods for data storage. Patterned media, ${ }^{1)}$,2) in which data are stored in isolated single-domain islands, have been one of the widely discussed possibilities for extending data densities with its capability to obtain higher storage densities well in excess of 1 Tbit/in ${ }^{2}$.

Pattern generation can be accomplished by several methods e.g. lithography, ${ }^{3)}$ Irradiation through a mask, ${ }^{4)}$ milling with focused ion beam ${ }^{5)}$ and imprint technology.6) In our present work, self-assembly configuration prepared by sputtering method is used because it has the potential to fabricate very fine-scale structures over large areas in a rapid process and no chemical used in the process.

The requirements of magnetic material for patterned media are high coercivity, chemical stability and perpendicular anisotropy. Hexagonal Strontium ferrite having the chemical formula of $\mathrm{SrO} \cdot 6 \mathrm{Fe}_{2} \mathrm{O}_{3}$ $(\mathrm{SrM})$ as well as hexagonal Barium ferrite $\left(\mathrm{BaO} \cdot 6 \mathrm{Fe}_{2} \mathrm{O}_{3}\right.$, $\mathrm{BaM}$ ) has fairly large magnetocrystalline anisotropy, excellent chemical stability and magnetic moment parallel to the caxis. In addition, the minimum stable grain size for SrM, computed for a storage time constant 10 years using the media stability criterion $K_{\mathrm{u}} V>$ $60 \cdot \mathrm{k}_{\mathrm{B}} T$, is about $8.8 \mathrm{~nm}$ that is achieved $1.6 \mathrm{Tbits} / \mathrm{in}^{2}$ with inter-dot distance of $10 \mathrm{~nm}$.

The Au film, which has FCC structure, is expected to be used as the underlayer for promotion of $c$ axis orientation of hexagonal ferrite layer. The misfit ratio between (111) plane of FCC Au and $c$-plane of HCP SrM is $2.1 \%$. It has also been reported that the $\mathrm{Au}$ islands structure can be formed on $\mathrm{SiO}_{2} / \mathrm{Si}$ substrate ${ }^{7)}$ and Corning glass at substrate temperature, $T_{\mathrm{u}}$, higher than
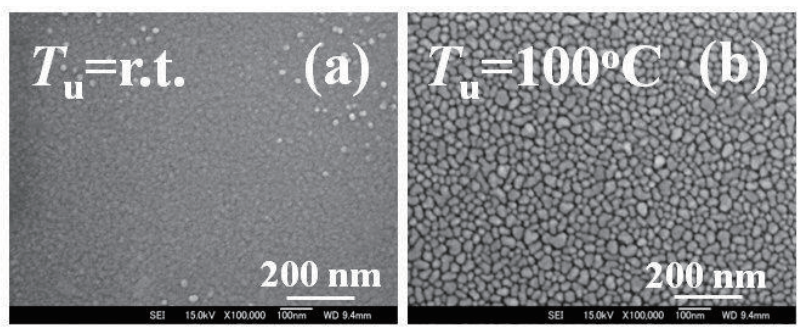

Fig. 1 SEM micrograph of Au surface at (a) r.t. and (b) $100{ }^{\circ} \mathrm{C}$.

$100{ }^{\circ} \mathrm{C}$ while the continuous films can be prepared at room temperature, r.t., as shown in Fig. 1 and the $\mathrm{Au}$ dot size decreases with increasing $T_{\mathrm{u}}{ }^{8)}$

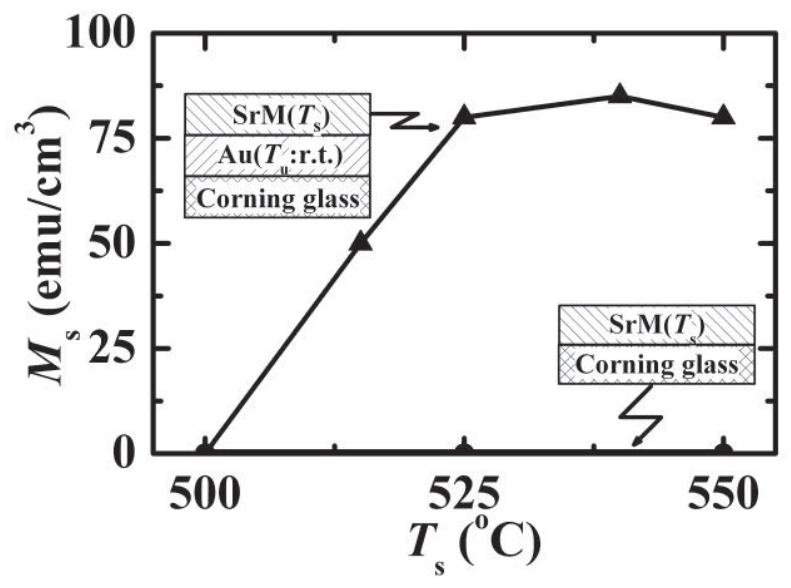

Fig. 2 Dependence of saturation magnetization, $M_{\mathrm{s}}$, of SrM thin films on substrate temperature, $T_{\mathrm{s}}$.

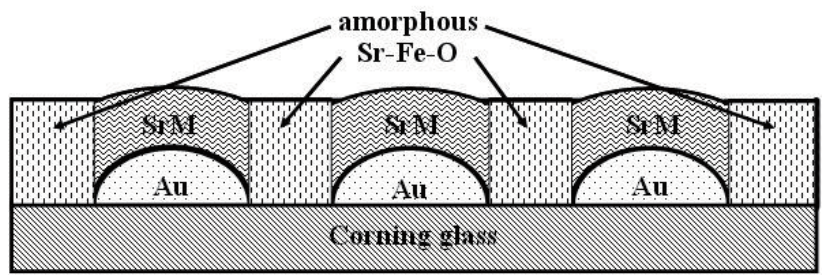

Fig. 3 Schematic of cross sectional view of partially crystallization of SrM. 
Figure 2 shows the dependence of saturation magnetization, $M_{\mathrm{s}}$, of $\mathrm{SrM}$ thin films on the substrate temperature, $T_{\mathrm{s}}$. The SrM thin films are amorphous at $T_{\mathrm{s}}$ below 550 and $515{ }^{\circ} \mathrm{C}$ for $\mathrm{SrM}$ thin films without underlayer and with $\mathrm{Au}$ underlayer, respectively. The $M_{\mathrm{s}}$ increases from zero to $80 \mathrm{emu} / \mathrm{cm}^{3}$ as substrate temperature, $T_{\mathrm{s}}$, was elevated from 500 to $525{ }^{\circ} \mathrm{C}$. Therefore, it is possible to get the isolated SrM grains on Au nano-dots prepared at $T_{\mathrm{s}}$ of $525{ }^{\circ} \mathrm{C}$. The proposed cross sectional structure in this study is schematically shown in Fig. 3.

\section{Experiments}

The SrM film was deposited on Au underlayer using a magnetron sputtering apparatus. The glass was used as substrate and thickness of SrM layer was 30 $\mathrm{nm}$. After evacuating the chamber to a pressure below $2 \times 10^{-6}$ Torr, argon (Ar) was introduced and sputtering pressure was set at $2.0 \mathrm{~m}$ Torr for Au underlayer film. In order to get $\mathrm{Au}$ island structure, the substrate

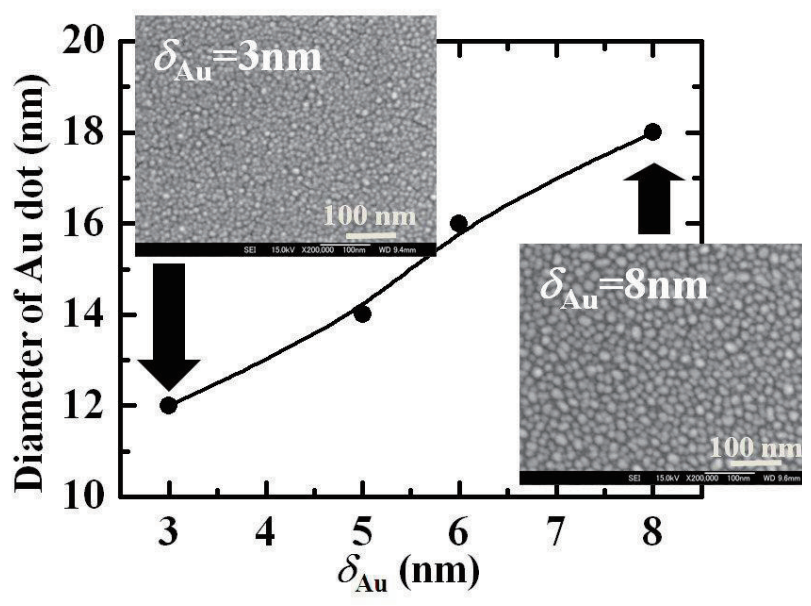

Fig. 4 Dependence of Au dot size on Au thickness.

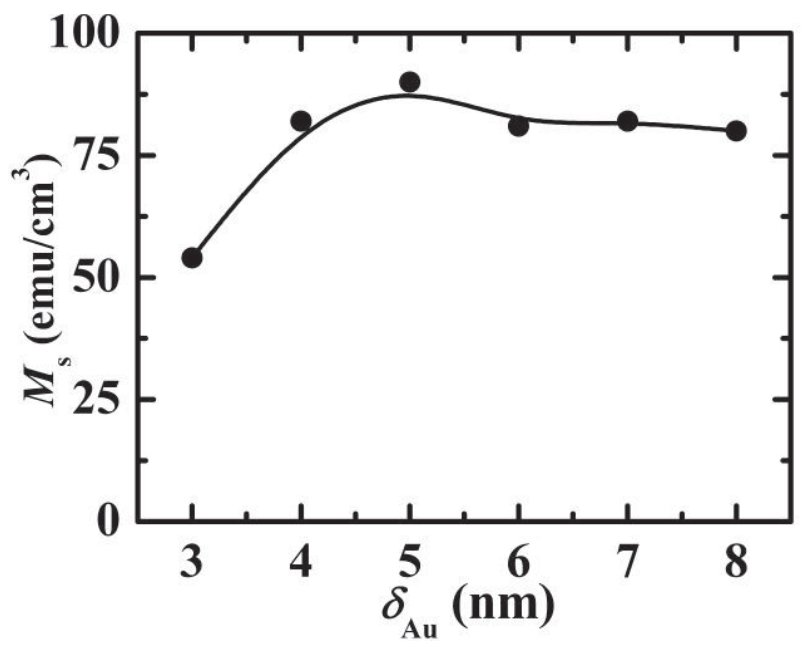

Fig. 5 Saturation magnetization, $M_{\mathrm{s}}$, of SrM thin films as a function of $\delta_{\mathrm{Au}}$. temperature for $\mathrm{Au}$ underlayer, $T_{\mathrm{u}}$, was kept at $300{ }^{\circ} \mathrm{C} .{ }^{8)}$ A sintered ferrite disk with stiochiometric composition of M-type, i.e. $\mathrm{Sr}: \mathrm{Fe}=1: 12$, was used as the target for $\mathrm{SrM}$ film. The mixture gas of $\mathrm{Ar}$ and $\mathrm{O}_{2}$ with gas pressure of 1.8 and 0.2 mTorr, respectively, was used as sputtering gas. The substrate temperature for SrM layer, $T_{\mathrm{s}}$, was kept constant at $525{ }^{\circ} \mathrm{C}$. The Au thickness was varied from $3-8 \mathrm{~nm}$. The crystal structures of the films were determined by x-ray diffractometer (XRD) with $\mathrm{Cu} \mathrm{K} \mathrm{K}_{\alpha}$ radiation. The magnetic properties were measured by vibrating sample magnetometer (VSM) with maximum field strength of $24 \mathrm{kOe}$. The surface morphologies of the films were observed by field emission type of scanning electron microscope (FE-SEM). The magnetic domain structures were observed by magnetic force microscope (MFM).

\section{Results and Discussion}

The Au dot size decreases with decreasing the thickness of Au underlayer, $\delta_{\mathrm{Au}}$, and its size is about 12 $\mathrm{nm}$ at $\delta$ Au of $3 \mathrm{~nm}$ as shown in Fig. 4 . So, the dot density and consequently the areal density of $\mathrm{Au}$ dot will be

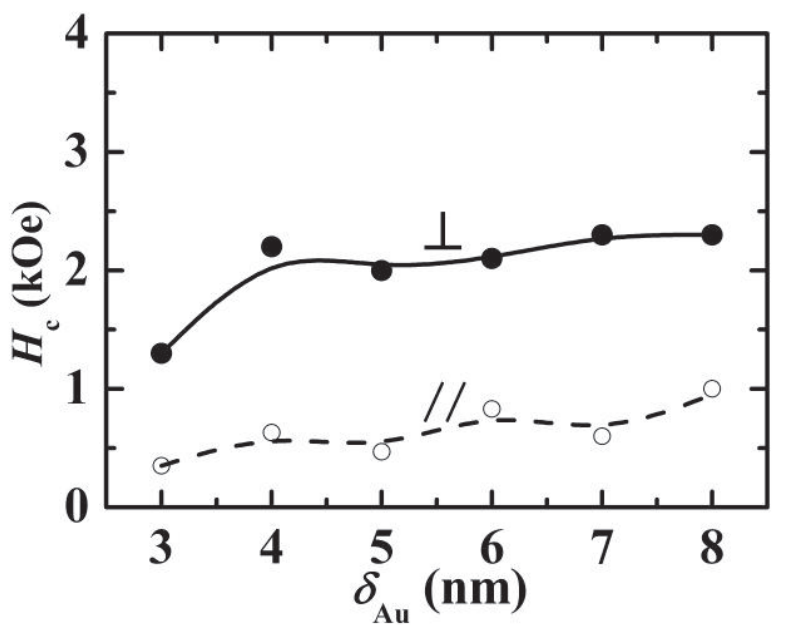

Fig. 6 Dependence of coercivity, $H_{c}$, of SrM thin films on $\delta_{\mathrm{Au}}$.

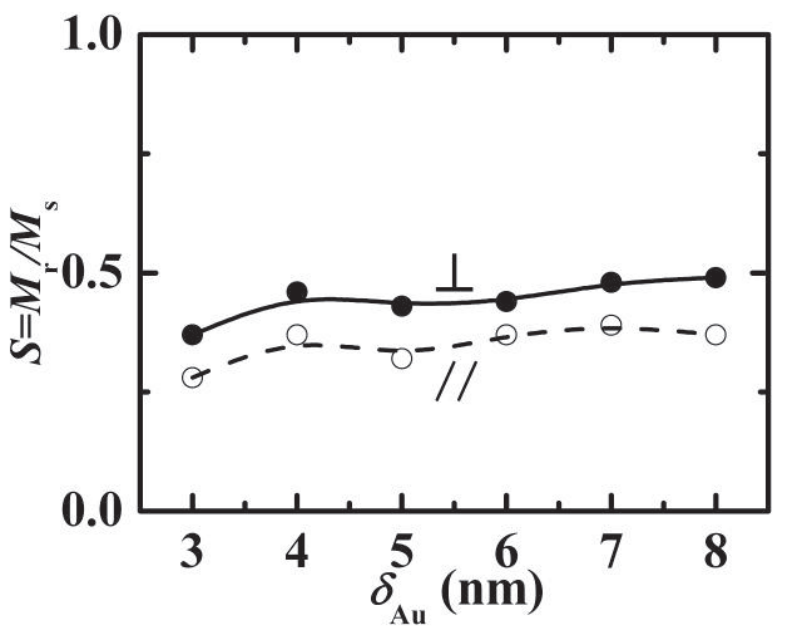

Fig. 7 Squareness ratio, $S$, of $\mathrm{SrM} / \mathrm{Au}$ thin films with various $\delta_{\mathrm{Au}}$. 
increased. From these results in order to achieve higher $\mathrm{Au}$ dot density and consequently higher density of SrM, an attempt to decreasing Au thickness has been carried out.

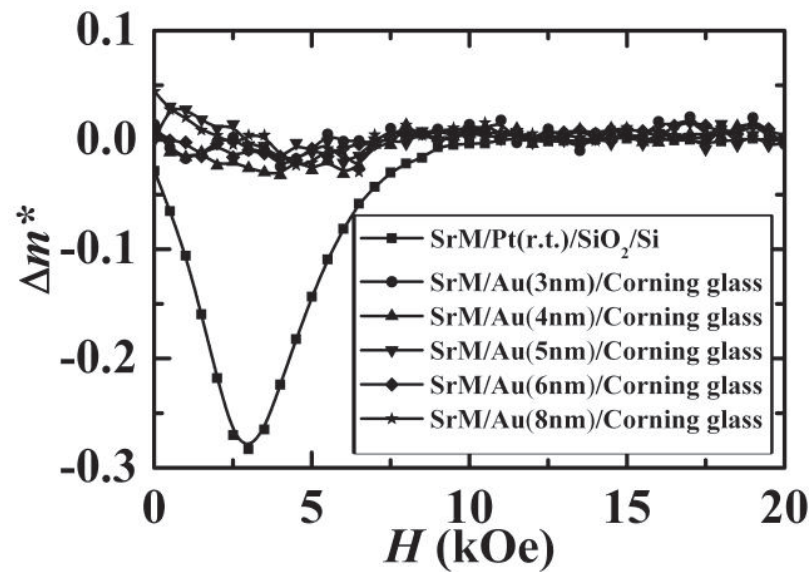

Fig. $8 \Delta m^{*}$ deviations for $\mathrm{SrM} / \mathrm{Au}$ thin films with different $\delta_{\mathrm{Au}}$.

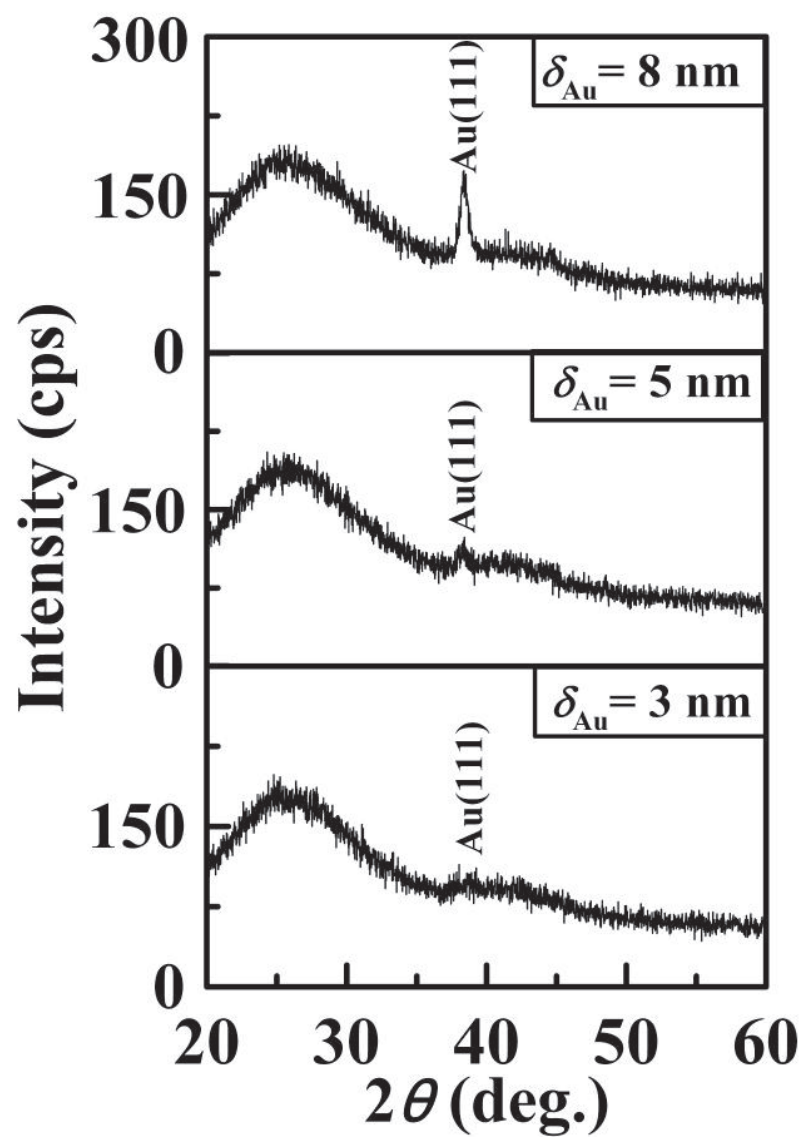

Fig. 9 XRD diagrams for SrM thin films on $\mathrm{Au}$ underlayer prepared at various $\mathrm{Au}$ thickness, $\delta_{\mathrm{Au}}$.

Figure 5 shows the saturation magnetization, $M_{\mathrm{s}}$, of $\mathrm{SrM} / \mathrm{Au}$ thin films as a function of $\delta$ Au. With decreasing $\mathrm{Au}$ thickness to $4 \mathrm{~nm}$, the $M_{\mathrm{s}}$ is almost constant at about $80 \mathrm{emu} / \mathrm{cm}^{3}$. All of these values of $M_{\mathrm{s}}$ are lower than that of the theoretical value of standard SrM film $\left(360 \mathrm{emu} / \mathrm{cm}^{3}\right)$. This might be due to non-magnetic phase existed in the space between the magnetic particles in SrM thin films. With further decreasing $\delta_{\mathrm{Au}}$, the $M_{\mathrm{s}}$ also decreases. It is contributed from decreasing degree of crystallization of SrM thin films.

Figure 6 shows the dependence of perpendicular and in-plane coercivity for the films on $\delta_{\text {Au }}$. The perpendicular coercivities, $H_{\mathrm{c} \perp}$, are higher than the in-plane coercivities, $H_{c / l}$. This means that the perpendicular anisotropy of SrM thin film. The $H_{\mathrm{c}_{\perp}}$ of $\mathrm{SrM}$ thin films on $\mathrm{Au}$ underlayer gradually increases and keeps almost constant at $2.3 \mathrm{kOe}$ with increasing $\delta_{\text {Au }}$ above $4 \mathrm{~nm}$. The $H_{\mathrm{c} / /}$ is also gradually increases with increasing $\delta$ Au to reach $1 \mathrm{kOe}$ at $\delta$ Au of $8 \mathrm{~nm}$.

The value of remanent squareness ratio, $S$, of $\mathrm{SrM}$ thin film on $\mathrm{Au}$ underlayer as a function of $\delta_{\mathrm{Au}}$ is shown in Fig. 7. The trend of $S$ is also as same as that for $H_{c}$. The $S_{/ /}$gradually increases and keeps constant when $\delta_{\mathrm{Au}}$ is above $4 \mathrm{~nm}$.

The interactions between magnetic particles of the thin films have been evaluated with the $\Delta m^{*}$ method.9) Following this method, the presence and strength of interactions is described as this equation:

$$
\Delta m^{*}(H)=m_{\mathrm{i}}(H)-0.5 \times\left[m_{\mathrm{u}}(H)+m_{\mathrm{l}}(H)\right]
$$

where $m_{\mathrm{i}}(H)$ is the initial magnetization curve, $m_{\mathrm{u}}(H)$ is the upper branch and $m_{1}(H)$ is the lower branch of the hysteresis loop. The finite negative and positive values of $\Delta m^{*}$ indicate the magnetostatic and exchange coupling, respectively.

In Fig. 8 the $\Delta m^{*}(\mathrm{H})$ deviations of the SrM/Au thin films with different $\delta_{\mathrm{Au}}$ are reported. It indicates negative interactions in the films. The peak of $\Delta m^{*}$ of $\mathrm{SrM} / \mathrm{Au}$ thin films is smaller in all films compared to $\mathrm{SrM} / \mathrm{Pt}$ that is the continuous film ${ }^{10)}$, which indicate a weaker interactions. From this results, it might be contributed from non-magnetic phase existed in the space between the magnetic particles in SrM thin films and hence the intergranular interactions is reduced.

Figure 9 shows the $x^{-r a y}$ diffraction diagrams of the film prepared on $\mathrm{Au}$ underlayer with various thicknesses of underlayer, $\delta_{\mathrm{Au}}$. The preferred (111) orientation of FCC lattice of Au underlayer has been detected in the XRD diagrams. The intensity of the diffraction line from $\mathrm{Au}(111)$ decreases with decrease of $\delta_{\mathrm{Au}}$. No obvious SrM peaks for all films were found.

Figure 10 shows surface morphology of $\mathrm{SrM}$ deposited on $\mathrm{Au}(111)$ underlayer with various $\delta_{\mathrm{Au}}$. From these surface images, it was found that the SrM surface structure corresponds to that of underlayer surface. The grain size of $\mathrm{SrM}$ decreases with decreasing $\delta_{\mathrm{Au}}$. 

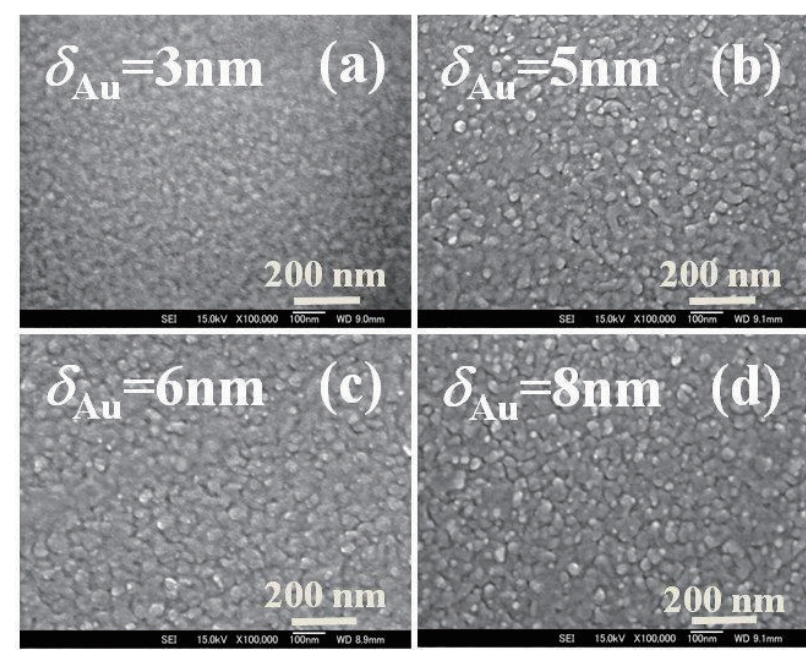

Fig. 10 Surface images of SrM thin films on $\mathrm{Au}$ prepared at various $\delta_{\mathrm{Au}}$.

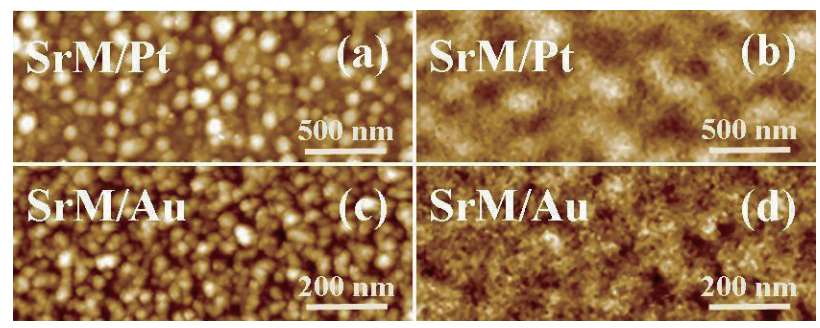

Fig. 11 (a) SrM surface and (b) SrM magnetic domain structure for Pt underlayer, (c) SrM surface and (d) SrM magnetic domain structure for $3 \mathrm{~nm}$ thick Au underlayer.

Figure 11 shows surface morphology and magnetic domain structure for $\mathrm{SrM} / \mathrm{Pt}$ and $\mathrm{SrM} / \mathrm{Au}$ thin films. The grain size of $\mathrm{SrM}$ deposited on $3 \mathrm{~nm}$ thick $\mathrm{Au}$ underlayer surface in Fig. 11(c) is less than that for Pt underlayer surface in Fig. 11(a). Observed magnetic domains shown in Fig. 11(b) are continuous with the length of over $400 \mathrm{~nm}$. On the other hand, the magnetic domains shown in Fig. 11(d) are obviously divided into small sizes, and the sizes are around $50 \mathrm{~nm}$ at the minimum that is less than that for Pt underlayer in Fig. 11(b). The reasons why the observed magnetic domain size in Fig. 11(d) are larger than the Au nano-dots is supposed to do with the resolution limit of the MFM, and spread of the leakage field from the domains.

\section{Conclusions}

SrM thin films can be prepared on $3-8 \mathrm{~nm}$ thick $\mathrm{Au}$ nano-dots at $T_{\mathrm{u}}$ of $300{ }^{\circ} \mathrm{C}$. With decreasing $\mathrm{Au}$ thickness, the dot density and consequently the areal density will be increased. Smaller magnetic domain size for SrM can be expected by reducing the Au nano-dots. The magnetic interaction between SrM grains prepared on $\mathrm{Au}$ nano-dots is reduced in compare with $\mathrm{SrM} / \mathrm{Pt}$ thin film that is the continuous film.

Acknowledgements This work was supported in part by Grants-in-Aid for Scientific Research (B) and Grant-in-Aid for JSPS Fellows from Japan Society for Promotion of Science (JSPS).

\section{References}

1) S. Y. Chou: Proc. IEEE, 85, 652 (1997).

2) R. L. White, R. M. H. New, and R. F. W. Pease: IEEE Trans. Magn., 33, 990 (1997).

3) F. J. Castano, Y. Hao, S. Haratani, C. A. Ross, B. Vogeli, M. Walsh, and H. I. Smith: IEEE Trans. Magn., 37, 2073 (2001).

4) C. Chappert, H. Bernas, J. Ferre, V. Kottler, J.-P. Jamet, Y. Chen, E. Cambril, T. Devolder, F. Rousseaux, V. Mathet, and H. Launois: Science, 280, 1919 (1998).

5) C. T. Rettner, M. E. Best, and B. D. Terris: IEEE Trans Magn., 37, 1649 (2001)

6) B. Heidari, I. Maximov, and L. Montelius: J. Vac. Sci. Tech. B., 18, 3557 (2000)

7) A. Kaewrawang, G. Ishida, X. Liu, and A. Morisako: IEEE Trans. Magn., 44, 2899 (2008).

8) A. Kaewrawang, A. Ghasemi, X. Liu, and A. Morisako: $J$. Phys.:Conf. Series., (accepted to publish)

9) S. Thamm, and J. Hesse: J. Magn. Magn. Mater., 154, 254 (1996).

10) A. Kaewrawang, A. Ghasemi, X. Liu and A. Morisako: $J$. Magn. Magn. Mater., 321, 1939 (2009).

Received Oct. 19, 2009; Revised Jan. 21, 2010; Accepted Mar. 17,2010 